Théologiques

Théologiques

\title{
Le problème du mal et du péché selon Nishitani
}

\section{Bernard Stevens}

Volume 20, numéro 1-2, 2012

Les philosophes de l'École de Kyōto et la théologie

URI : https://id.erudit.org/iderudit/1018859ar

DOI : https://doi.org/10.7202/1018859ar

Aller au sommaire du numéro

Éditeur(s)

Faculté de théologie et de sciences des religions, Université de Montréal

ISSN

1188-7109 (imprimé)

1492-1413 (numérique)

Découvrir la revue

Citer cet article

Stevens, B. (2012). Le problème du mal et du péché selon Nishitani.

Théologiques, 20(1-2), 199-213. https://doi.org/10.7202/1018859ar

\section{Résumé de l'article}

Il s'agit d'un exposé de la manière dont Nishitani présente la question du mal et du péché dans Qu'est-ce que la religion ?, tâchant de montrer que cette question joue un rôle essentiel dans l'approfondissement de la prise de conscience en direction du néant et de la vacuité. Afin de mieux mettre en perspective la pensée de Nishitani, celle-ci est brièvement comparée à celle de Paul Ricoeur à propos de la même problématique. La mise en relief de leurs positions métaphysiques divergentes permet de constater que le « penser plus et autrement ", qu'induit l'aporie du mal, les conduit à des positions finalement très proches. 


\title{
Le problème du mal et du péché selon Nishitani
}

\author{
Bernard STEvens" \\ Philosophie \\ Université Catholique de Louvain (Belgique)
}

Au cours de la réflexion entreprise par Nishitani dans sa quête du religieux, au croisement du bouddhisme et du christianisme, de nombreuses notions de la théologie catholique et protestante sont réexaminées sous un éclairage nouveau: par exemple, le Dieu personnel est repensé à partir de l'épreuve de la vacuité (空, $k \bar{u}$ ), sous l'éclairage réciproque de la kénose et du non-ego (無我, muga); l'histoire du salut est confrontée à la vision du kalpa (l'aiōn bouddhique); l'amour (agapè) est revitalisé au contact de la compassion (karuna), etc. Et plusieurs thématiques communes aux deux religions sont méditées, sans que l'on sache toujours exactement d'où provient l'inspiration dominante: la souffrance, la conversion, la mort et la nouvelle naissance... Les auteurs chrétiens que cite Nishitani sont légion, de saint Paul à Karl Barth, en passant par pratiquement tous les moments les plus décisifs de la spiritualité chrétienne (Augustin, Maître Eckhart, Luther, François d'Assise...), beaucoup moins, toutefois, les hauts lieux de la spéculation (Thomas ou Hegel, par exemple, ne sont jamais conviés). Cependant, étant donné la fascination manifeste de Nishitani pour des auteurs tels que Heidegger et surtout Nietzsche, cités à presque tous les chapitres, on pourrait croire que sa pensée religieuse serait tentée de se déployer «par-delà le bien et le mal», dans une sorte de contemplation

* Bernard Stevens est professeur et chercheur à l'Université Catholique de Louvain (Louvain-la-Neuve). Il tente, dans ses derniers travaux, de mieux faire connaître la philosophie japonaise contemporaine au lectorat francophone et d'établir un pont entre la phénoménologie et la pensée de l'École de Kyōto. Il est actuellement occupé à traduire Qu'est-ce que la religion? de Nishitani. Derniers ouvrages parus: (2005) Une invitation à la philosophie japonaise. Autour de Nishida, Paris, CNRS et (2009) Le nouveau capitalisme asiatique. Le modèle japonais, Louvain-la-Neuve, BruylantAcademia.

(C) Revue Théologiques 2012. Tout droit réservé. 
mystique extatique, évoluant dans l'innocence du devenir et l'absence de préoccupation morale. Or, il n'en est rien. La question du mal et du péché joue un rôle déterminant dans le mouvement d'ensemble de sa démarche et c'est ce que nous aimerions mettre ici en évidence. Nous limitant ici à Qu'est-ce que la religion? ${ }^{1}$, nous allons voir comment apparaissent ces notions et quelle est leur fonction dans le déploiement de l'expérience de la pensée proposée par Nishitani. Il nous a semblé en conclusion qu'une brève confrontation avec son contemporain chrétien, Paul Ricœur, pouvait jeter un éclairage précieux sur le propos.

\section{Lecture de Qu'est-ce que la religion?}

Soulignant le caractère impérieux de la religion — elle est «le problème cardinal» de notre vie (大問題, dai mondai), puisqu'elle concerne «la question de savoir si nous terminerons notre existence dans la perdition ou bien si nous obtiendrons la vie éternelle» (4) —, Nishitani évoque quelques-unes des situations dans lesquelles on peut percevoir, par-delà toute approche utilitariste, l'exigence religieuse: «la mort, le néant ou le péché $^{2}$ (6), dit-il, sont des événements qui, lorsque nous y sommes confrontés, creusent en nous un abîme de non-sens et d'interrogation qui est la première source de la quête religieuse. Ces expériences extrêmes, qui évoquent les Grenzsituationen de Karl Jaspers, permettent d'effectuer un 《pas en arrière» (退歩, taiho) par rapport à l'affairement quotidien et elles nous confrontent à une réalité qui est en vérité toujours déjà là: la béance du néant fondamental sur lequel est bâtie notre vie. Avec la prise de conscience d'une telle béance, ce qui se produit c'est l'éveil à soi de notre existence (自己存在の自覚, jiko sonzai no jikaku), occasionnant, en une véritable conversion, le décentrement de notre attention par rapport à notre égocentrisme habituel, pour nous rendre alors plus attentifs au centre inapparent de la giration des choses et de nous-mêmes (individu et humanité). Ce à quoi la religion donne accès, poursuit Nishitani, c'est «l'éveil à soi réel de la réalité ${ }^{3} »(8)$ : la réalité advient pleinement à soi, dans une prise de conscience qui est désormais délestée des obstructions édifiées par

1. 西谷啓治, 宗教とは何か, 創文社 1961. À partir d'ici, toutes les références à cet ouvrage seront simplement signalées au moyen d'un chiffre entre parenthèses.

2. 死とか虚無とか或は罪, shi toka kyomu toka aruiha tsumi.

3. 実在の実在的な自覚, jitsuzai no jitsuzaitekina jikaku. On pourrait également dire: «la prise de conscience réelle de la réalité », mais serait alors perdue la notion d' «éveil» présente dans l'idéogramme 覚, kaku. 
l'ego solipsiste, celui que la modernité a poussé à l'extrême, dans la foulée du cogito cartésien. Pour mieux saisir cela, précise Nishitani, il faut comprendre dans la notion de réalité, non pas l'extériorité objective des choses du monde, ni l'intériorité subjective des actes de conscience, mais une qualité de venue en présence qui est par-delà (ou en deçà de) toute dichotomie sujet-objet, par-delà toute opposition intérieur-extérieur, par-delà le plan de la conscience (意識の場, ishiki no ba) qui fonde de telles dichotomies et contradictions: une qualité où les choses du monde et le soi viennent conjointement en présence, sont une même venue en présence, une même vie animant indistinctement tout ce qui est, en une co-respondance proprement universelle.

Pour atteindre ce plan nouveau, Nishitani nous explique qu'il faut commencer par pouvoir nous affranchir du plan du «je pense donc je suis », enfermé dans son évidence à soi oblitérante. Et il ajoute que c'est l'attachement à ce plan qui crée "par exemple le problème de l'égoïsme, ou celui du bien et du mal dans la nature humaine, le problème du mal radical et du péché, le problème de la solitude ou de la perte de soi dans la société, le problème de la possibilité de la connaissance, les exigences de la rédemption ou de la délivrance» (20). En somme, les difficultés éthiques et religieuses fondamentales surgissent de ce plan de l'ego représentant et voulant: elles sont liées au mode d'être d'un soi égocentrique se cramponnant à soi. À ce niveau, l'ego, aveuglé par l'évidence du cogito, a perdu le contact avec sa nature la plus propre, dans sa radicalité première. Lorsque ce plan devient consciemment problématique, notamment par l'épreuve des situations limites, notamment par la prise de conscience du néant ou encore, celle de l'être pécheur, le doute (疑う, utagau) qui s'installe alors peut nous conduire bien au-delà du plan théorique propre au doute méthodique et nous mettre sur la voie existentielle et religieuse du "grand doute » (大疑, taigi) - le doute qui englobe le soi et toute chose dans une immense interrogation, couvrant l'ensemble de la réalité: c'est la réalité elle-même alors qui est doute et qui engloutit le soi dans ce doute, au point de faire conjointement l'expérience de «la grande mort» (大死, taishi). Celle-ci devient alors le site de la possible conversion, de la mort débouchant sur la nouvelle naissance.

Resserrant encore le propos autour de ce grand doute, Nishitani insiste pour dire que ce ne sont pas seulement la mort et le néant qui y donnent 
accès, mais bien aussi «le mal» (悪, $a k u$ ) et «le péché»(罪, tsumi $)^{4}$. Et il ajoute que ces derniers sont des exemples privilégiés pour comprendre comment transcender le plan de la conscience. Au niveau de ce dernier, en effet, lorsque l'on reste rivé à la représentation, on parle du «je» qui commet «le mal», comme si l'agent et le mal commis étaient deux choses séparées. Or l'actualisation réelle du mal et du péché se produit lorsque le plan de la conscience représentative est transcendé vers une dimension plus profonde, celle dont Kant a eu l'intuition lorsqu'il a parlé du «mal radical» (根元悪, kongen aku), enraciné au fond du sujet: à la fois agi par le sujet, mais antérieur à lui. Par-delà le mal commis, qui est perçu comme un événement temporel du monde de l'expérience, il y a une dimension qui le précède, non pas chronologiquement, mais de manière en quelque sorte «transtemporelle». Le mal commis — dans cet «instant» qui, selon Kierkegaard, est un "atome d'éternité» — s'enracine dans un sol de réalité plus vaste et insaisissable qui fonde le sujet et l'inscrit dans une condition qu'il partage avec ses semblables. De sorte que lorsque l'individu commet le mal et qu'il en prend conscience, ce qui se produit c'est l'éveil à soi du mal en son ainsité, en tant que réalité. Le christianisme, poursuit Nishitani, a tenté de dire cette nature à la fois préexistante et collective du mal au moyen de la notion de «péché originel ( (原罪, genzai) ou de «faute héréditaire» (宿罪, shukuzai) et le bouddhisme a développé l'idée du «karma» (業, $g \bar{o})$ et de «l'ignorance» (無明, mumyō). Ce sont chaque fois des tentatives d'exprimer l'appropriation par le soi individuel d'une réalité pécheresse qu'il partage déjà toujours avec toute l'humanité. Ce dépassement du plan de la conscience représentative vers le plan de la réalité englobante que s'approprie le soi, indique le passage de l'éthique philosophique à la conscience religieuse - tout comme lorsque le doute méthodique se transforme en grand doute.

Nishitani est proche ici de Karl Barth lorsqu'il met en relief la nature intégralement pécheresse de l'homme et qu'il souligne en outre que ce dernier n'a d'autre possibilité, pour s'en affranchir, que d'y consentir en la confessant et en prenant conscience de sa totale corruption comme de sa totale impuissance (sans même espérer s'accrocher à un Anknüpfungspunkt, un "point de contact» avec Dieu, formel ou rationnel, comme le voulait Emil Brunner). Cette prise de conscience du péché en tant que "grande réalité»(大きなリアリテイ, ôkina rialitei), ajoute-t-il, est une véritable

4. P. 27 et encore p. 104 et 106 où c'est le «péché originel» (原罪, genzai) qui est mis en évidence. 
néantisation par laquelle la béance du néant qui fonde l'homme se révèle pleinement à lui. C'est là que peut alors s'ouvrir le lieu d'accueil pour l'amour salvateur de Dieu.

Le salut de l'homme se produit tout en restant dans le mal. La capacité de faire le mal est le signe du néant au fondement d'un être créé par Dieu. Et parce que ce Dieu est omnipotent, il a le pouvoir de pardonner le mal et de sauver le pécheur, quand celui-ci se tourne vers Lui dans la foi. La rédemption divine, au travers du "second Adam ", peut sauver la condition déchue de l'homme qui s'est manifestée dans le péché du "premier Adam ». Cette rédemption s'inscrit dans l'ouverture créée par la béance du néant propre à la condition humaine pécheresse. Cette ouverture ellemême est déjà l'œuvre divine, rendue possible par la dissolution de l'ego à même l'éveil à soi de la réalité en tant que révélation du mal radical en son ainsité. Le fait d'accueillir l'amour salvateur est ce que l'on appelle la conversion de la foi. Mais cette dernière n'est pas un acte de l'individu qui choisit de croire en Dieu: venant, tout comme le mal, de plus loin que lui, elle est une expression de la grande réalité. Elle peut être comprise comme une grâce rédemptrice que Dieu accorde, par l'action du Saint-Esprit. Et Nishitani joint ici à la thématique paulinienne de la grâce celle, amidiste, du transfert de mérites par le vœu originel du Bouddha Amida. Mais en retour, ajoute-t-il, il n'y aurait pas de vœu du Bouddha sans la foi fervente du pratiquant: l'homme et le divin sont unis (Amida est ici assimilé au divin). "Celui qui s'unit au Seigneur n'est avec lui qu'un seul esprit» (1 Co $6,17)$. Or, cette expérience religieuse de la foi (bouddhique ou chrétienne) est celle où le soi devient le plus réellement et le plus intimement lui-même, dans son unicité - à la différence de l'ego cartésien, qui est universalisable et, de ce fait, interchangeable avec tout autre. En elle, se produit l'actualisation de la réalité dans sa prise de conscience appropriante: la conversion de la négation absolue en affirmation absolue est l'actualisation de la grande réalité. Et c'est sur ce plan que la résolution chrétienne de la foi équivaut à "l'obtention instantanée de la renaissance» (即得往生, sokutoku oj $j \bar{o}, 34)$ pour celui qui s'en est remis pleinement au Bouddha-Amida.

L'amour divin, souligne bien notre auteur, n'est pas identique à l'amour humain. Dans son amour, en effet, le Christ apporte «le glaive» (Mt 10,34). C'est le glaive qui, en tranchant la mort spirituelle qui est dans le péché, nie le soi égocentrique de l'homme. Et la nouvelle naissance, aussi bien que l'amour du prochain, inspirés de Dieu (神の霊感を受け, kami no reikan o uke), devront agir selon le même souffle (息吹き, ibuki), celui que le Saint-Esprit (聖霊, seirei) nous a inspiré (息吹かれた, ibukareta, 33 et 
48). Or, l'amour du prochain est, comme la compassion bouddhique, « un amour indifférencié pour ses amis et ses ennemis ", c'est un amour inspiré de l'amour de Dieu qui, pour prendre en Christ la forme du serviteur, s'est vidé de sa forme divine (kénose) et qui, parce qu'il n'a pas d'attachement à soi ni d'égoïsme, pardonne même au malfaiteur qui s'est tourné contre Lui. Ce type d'amour est propre à une personnalité divine que Nishitani aime qualifier de "non-personnelle » lorsqu'il cherche à en cerner le caractère. Il est fondé dans le non-ego, animé par la «vacuité» (空, $k \bar{u}$ ), d'où procèdent la grande compassion et la grande sagesse. La personnalité d'un Dieu ainsi comprise, inhabituelle dans le christianisme, n'y a été entrevue que par la théologie négative, dont Maître Eckhart est le plus éminent exemple, cité à maintes reprises par le penseur japonais (en particulier p. 70ss).

On peut voir ainsi que la manière dont Nishitani exprime les convictions relatives à la thématique du péché et du mal est marquée par la perspective de la vacuité (空, $k \bar{u}$ ), ou plus précisément: par la perspective où le néant est transcendé par la vacuité. Or, lorsque cette vacuité vient éclairer le sens proprement occidental et judéo-chrétien de l'histoire, elle donne une coloration propre au récit de la chute et de la rédemption: au passage de la «mort» à la «vie». Toute cette réinterprétation a lieu au sein d'une conception mahāyāniste où le nirvāna se produit à même le samsāra, où la ronde de souffrance et de mort peut être transfigurée par l'extinction de l'avidité et de l'ignorance ainsi que de l'attachement à soi qui s'y trouve lié. On peut en prendre conscience, individuellement, lors du «lâcher prise de corps et d'esprit ", celui qu'induit notamment la pratique du «juste s'asseoir» (座禅, zazen) $)^{5}$, nous libérant des désirs mondains et des passions. L'esprit est alors désentravé de ses empêchements, il dépasse les obscurcissements de l'ignorance et s'ouvre à la limpidité du monde. Vue depuis cette perspective, l'histoire chrétienne de la chute est une histoire qui, en termes bouddhiques, est encore immergée dans l'ignorance et animée par le devenir sans fin de l'activité karmique. Mais alors que le devenir temporel bouddhique est cyclique, l'histoire judéo-chrétienne est téléologique. Cette dernière est liée à la conscience de soi de l'homme occidental en tant qu'être libre et pécheur: le sens de l'histoire, centrée sur l'homme, est déterminé par la manière dont l'homme assume cette conscience et cette condition. Le sens de l'histoire sera, collectivement, celui du salut de

5. Il s'agit de l'expression de Dōgen, abondamment commentée par Nishitani à la page 208. 
l'homme pécheur et, individuellement, celui du choix en faveur ou non de ce salut. Et comme le salut est octroyé par Dieu, la direction téléologique de l'histoire en devient une eschatologique. Le commencement et la fin de l'histoire sont déterminés respectivement par la chute du premier Adam et le retour du Christ ou, en d'autres mots, par la punition divine et par le jugement final. Nishitani souligne longuement que la dimension manifestement mythique de cette vision eschatologique de l'histoire doit pouvoir être perçue autrement, dans une profondeur «transhistorique ", où le salut vient s'établir, non pas dans un futur lointain, mais dans l'immédiateté de l'ici-bas. Peut-être les notions nietzschéennes de l'éternel retour et de "l'innocence du devenir» (Unschuld des Werdens) nous y aideront-elles (236ss).

L'éternel retour rejoint le kalpa bouddhique en ce qu'il est une finitude infinie, un cercle tournant sans fin et "en vain» (umsonst). Mais dans le bouddhisme, la succession infinie des retours s'inscrit dans une juxtaposition infinie des kalpas coexistants, en une sorte de maintenant illimité: " une ouverture infinie " s'éclôt au fond du temps, comme la vacuité bleue du ciel. Chaque maintenant, suggère Nishitani, y est unique et met en évidence son ambiguité. L'ambiguiité du temps apparaît ici sous un aspect de nouveauté et d'impermanence - que Nishitani va s'employer à expliciter dans le sillage de la pensée heideggérienne. La nouveauté du temps, manifestant à son tour une ambiguïté, signifie une possibilité infinie qui est une liberté créatrice infinie; mais en même temps, du fait de cette dernière, elle signifie une poussée sans fin vers l'avant, un projet (Entwurf), qui devient comme un fardeau, comme une dette (Schuld) qu'il nous faut payer à l'infini (chaque paiement de dette est une nouvelle action qui, à son tour, a des conséquences, crée une nouvelle dette, selon la loi du karma). Bref, c'est un devenir incessant. Cette poussée infinie du temps mondain, «depuis les temps les plus reculés sans commencement ni fin », une poussée qui est animée par l'avidité, tout aussi infinie, est le sens véritable du mythe de la transmigration. Or, le temps, rappelle Nishitani, est également impermanence. Cette dernière, elle aussi ambiguë, signifie à la fois la volatilité du temps (chaque instant disparaît comme un éclair) et la négation de la permanence: elle est la liberté de pouvoir glisser sans être retenu par les restrictions des existences précédentes. C'est ainsi que l'ouverture infinie au fond du temps comporte elle-même globalement l'ambiguïté d'être à la fois le néant et la vacuité. C'est bien cette ambiguïté qui en fait le site éminent de la possible conversion, de la metanoia. 
Le surgissement du temps, ainsi décrit, s'accompagne, en effet, d'une ouverture infinie qui est par-delà le temps. Cette ouverture est un plan de néant qui s'étend directement sous nos pieds. L'agir constant, sous l'effet de la poussée continue, est en même temps totalement libre: notre existence y reçoit perpétuellement une détermination entièrement nouvelle. Par-delà la causation karmique continue, la nouveauté de chaque acte représente une liberté. L'impermanence signifie une non-permanence, c'està-dire la constante possibilité d'une libre création, sise dans le présent. Ainsi l'ouverture infinie est-elle la transcendance ek-statique de la liberté propre. Le néant apparaît ici comme le trait d'union entre le destin et la libre volonté, de sorte que la joie de la liberté est teintée de la tristesse du désespoir de la condition humaine. Mais comme le surgissement de cela provient du néant, il provient en somme du champ de la non-forme: là où la forme de la condition humaine est rejetée. C'est un surgissement d'être qui est commun à tous les vivants et qui est ontologiquement préalable à toute conformation humaine précise et à toute individualité. Le rapport aux autres ici n'est plus seulement celui de la causalité propre à la codépendance karmique, mais il est celui du rassemblement de l'être, propre à la co-présence. Le plus extrême égocentrisme débouche ainsi, depuis ce centre, sur la plus profonde intercommunication avec les autres. Le lien du destin se confond avec la liberté déliée d'un «soi qui n'est pas un soi ", et qui nie la volonté propre, s'oppose à la poussée infinie de l'avidité. L'égocentrisme de l'attachement à soi s'est transformé en la centralité du soi en relation d'interconnexion réciproque avec tous les autres. Et le soi au centre des choses qu'il rassemble est en même temps un soi qui consent aux autres, qui se décentre au profit de tous les autres. Le soi est le centre d'un recueillement des choses qui, chacune, à son tour, est le centre auquel le soi est subordonné.

Le faire ici, qui surgit de la vacuité, est un non-faire. Le cercle infernal de l'agir sans fin se transforme en un simple demeurer là. L'action prend la configuration du jeu, joué avec le sérieux d'un enfant - et en écho avec le grand jeu du monde évoqué par Héraclite. Elle est sans telos, elle est "sans pourquoi » (ohne warum). Elle est «autotélique », pure spontanéité. Elle n'est plus régie par un ego voulant et ignorant. L'agir, qui est ainsi non-agir, n'alimente plus la dette et cette dernière finit par être remboursée et dissoute. Elle est remplacée par la tâche ou la vocation, ainsi que par la responsabilité pour autrui, à son tour infinie. Le destin s'est retourné en vocation. On n'est plus endetté, mais redevable à autrui de sa propre essence. Le destin égocentrique de la pulsion infinie est remplacé par un 
être pour autrui, un allocentrisme. Et les autres, les choses du monde et le monde tout entier sont le centre de notre vie et le maître de notre action: chacun se subordonne à l'altérité. Le soi qui n'est pas un soi, qui est décentré de soi vers les autres, est le soi authentique, dans sa spontanéité originelle. Le soi et autrui se déploient dans un état de non-dualité entre soi et autrui - dans un état de mutuel amour (愛, ai) et de compassion (慈悲, jihi). C'est l'état de communion affective exprimé par le Cantique au soleil de François d'Assise, le personnage sur l'évocation duquel se clôt le beau livre de Nishitani.

\section{En guise de conclusion: une confrontation avec Paul Ricœur}

On voit ainsi comment, dans sa méditation sur le péché et le mal, la conversion et la nouvelle naissance, Nishitani croise dans un tissage unique les motifs chrétiens et bouddhiques et ce, en un discours où la tournure poétique du propos semble parfois l'emporter sur la provenance théologique des thèmes. La transformation intime occasionnée par le renversement du néant en vacuité permet, non pas de remplacer ou corriger les motifs chrétiens, mais au contraire de les penser à neuf et, peut-être, plus aisément. C'est un éclairage réciproque du bouddhisme et du christianisme qui se produit afin de clarifier la condition humaine, en l'occurrence dans sa dimension pécheresse. Et si on voulait confronter Nishitani au penseur chrétien, contemporain de lui, qui lui est au départ le plus opposé - je songe à Paul Ricœur - on constaterait que le passage par l'épreuve de la faillibilité humaine les rapproche au final et réduit leur différence, voire fait converger leurs parcours respectifs.

Le choix de Ricœur pour ces (trop elliptiques) considérations conclusives me semble particulièrement approprié. La question du mal est en effet centrale chez le philosophe français (c'est la source de sa "greffe» de l'herméneutique sur la phénoménologie) et sa perspective métaphysique propre établit un cadre qui, dans une apparente opposition à Nishitani, permet grâce à ce contraste de mieux saisir les enjeux de la confrontation bouddhisme-christianisme. Quand nous parlons de la «perspective métaphysique» de Ricœur, nous voulons dire deux choses. Il s'agit premièrement de son choix pour «l'affirmation originaire", qu'il place au fondement de l'ontologie, par opposition à tous les philosophes qui veulent faire de la négativité le ressort de leur réflexion (voir en particulier Ricœur 1955): il songe singulièrement à Sartre, mais peut-être aurait-il également classé ici Nishitani, s'il avait eu connaissance de son œuvre. La perspective métaphy- 
sique de Ricœur signifie, en deuxième lieu, le fait que le philosophe français se revendique, à maintes reprises, de la tradition "réflexive» qui a donné, en particulier, le kantisme et la phénoménologie de type husserlien (voir Ricœur 1969 et d'autres chapitres programmatiques du même ouvrage). Or, la philosophie réflexive remonte au cogito cartésien dont on sait désormais que Nishitani se donne beaucoup de peine à le déconstruire.

La thématique du mal, Ricœur l'explore à travers trois ouvrages majeurs: L'homme faillible (1960), La symbolique du mal (1960) et Le conflit des interprétations (1969). Plutôt que de le suivre à travers ce parcours complexe, nous allons utiliser un petit livre, tiré d'une conférence prononcée à Lausanne en 1985, Le mal (Ricœur 2004), dans lequel il rassemble les grandes lignes du problème.

Le mal présente un défi aussi bien à la théologie qu'à la philosophie et devient, face à l'échec apparent de la réflexion qui s'y manifeste, "comme une invitation à penser moins ou une provocation à penser plus, voire à penser autrement» (Ricœur 2004, 19). Tout comme Nishitani le met en évidence à propos des expériences extrêmes, et particulièrement celles du péché et du mal, Ricœur souligne que ce dernier met en question notre mode de penser soumis à l'exigence de la cohérence logique. Les théodicées, dont celle de Leibniz est le paradigme, ont tenté d'intégrer le mal dans un système ontothéologique où il se trouve au total justifié par des arguments spécieux, permettant de tenir ensemble les trois propositions: «Dieu est tout-puissant; Dieu est absolument bon; pourtant le mal existe». Aussi bien Nishitani que Ricœur rejettent les théodicées - y compris sous leurs avatars dialectiques —, car elles oblitèrent la réalité ténébreuse du mal.

Il faut remettre en évidence cette dernière, dit Ricœur, avant de s'engager dans une tentative de solution ou de réponse. Il souligne tout d'abord qu'en Occident nous réunissons sous un même terme des réalités différentes: le mal «commis» (le péché ou mal moral) et le mal «subi» (la souffrance). Or il est intéressant de noter ici que, même si en japonais la notion de mal (悪, $a k u$ ) est clairement du registre moral, au même titre que le péché ou l'impureté (罪, tsumi), Nishitani en parle dans un contexte où elle est perçue ensemble avec des expériences subies, telles que la maladie ou la mort, car elles ont toutes en commun avec le mal commis d'être une révélation de la béance du néant (虚無, kyomu). Cependant, à aucun moment il ne souligne en cette dernière la dimension du souffrir (苦しむ, kurushimu), mais bien celle, abyssale, du non-sens (無意味, mu.imi). Sans doute est-ce là une expression de la conception bouddhique selon laquelle la condition déchue de l'humain remonte, en premier lieu, à l'ignorance 
(無明, mumyō, correspondant à l'avidyā sanscrit). En tout état de cause, on perçoit ici, à même les vocables utilisés, le basso ostinato du négatif: 無, $m u$, en contraste avec "l'affirmation originaire", clamée par le philosophe français.

Le mal moral, poursuit Ricœur, peut être imputé à un agent responsable et susciter le blâme, alors que la souffrance est quelque chose qui affecte l'individu, sans qu'il en soit a priori responsable, et elle suscite la lamentation. Or, c'est bien conjointement sous ces deux aspects que théologie et philosophie cherchent à penser le mal comme leur racine commune. C'est que les deux phénomènes sont enchevêtrés. Au mal commis, on répond par la peine infligée. Et plus largement, une des causes principales de la souffrance est bien la violence, le mal, exercés par les humains entre eux. Le mal commis par l'un trouve sa réplique dans le mal subi par l'autre et il intensifie la lamentation. Nous découvrons ainsi une réalité où les deux versants du mal pointent vers l'unité de la condition humaine, dans une dimension d'énigme et d'opacité où les discours pour l'exprimer prennent une tournure symbolique ou mythique, nécessitant alors l'art de l'herméneute. Dans la culpabilité, se révèle un sentiment de dépendre de forces supérieures qui donne au coupable l'impression d'être lui-même victime. Et comme une peine infligée est une souffrance, la condition souffrante elle-même apparaît comme une punition collective ou individuelle, rejoignant l'obscurité des forces du mal. C'est ici qu'interviennent les symboles religieux et les mythes que Ricœur a examinés ailleurs pour le contexte judéo-grec et que Nishitani a évoqué au croisement du bouddhisme et du christianisme. Pour l'un comme pour l'autre, le mythe adamique joue un rôle clé. Et pour l'un comme pour l'autre, «le symbole donne à penser ", c'est-à-dire qu'il est un défi face à la tentation de renoncer à comprendre.

Le mythe adamique relate l'origine de la condition souffrante de l'homme dans la désobéissance humaine. Ce sera une des principales sources de la doctrine du péché originel, élaborée par Augustin. Ce dernier précise en outre la doctrine de la creatio ex nibilo qui place une dimension de néant au cœur de la création et permet de mieux comprendre comment le mal en tant que défaut d'être a pu surgir en son sein, trouvant sa plus forte expression dans la liberté d'un humain, destiné au bien, mais tenté par le mal, capable de "décliner» loin de Dieu. La question est devenue: «d'où vient que nous faisons le mal ? " et la réponse est dans une volonté mauvaise, un péché qui est nibil privativum, et dont la chute est responsable. Augustin introduit ainsi «la vision morale» du mal où celui-ci est 
soit un péché, soit une peine (individuelle ou collective). La souffrance est justifiée par la rétribution et celle-ci trouve son explication dans la dimension supra-individuelle (historique, générique) du péché originel. Le commentaire de Ricœur rejoint ici celui de Nishitani lorsqu'il explique que cette doctrine cherche à rendre compte de «l'expérience à la fois individuelle et communautaire de l'impuissance de l'homme face à la puissance démonique d'un mal déjà là, avant toute initiative mauvaise assignable à quelque intention délibérée » (2004, 37). Il s'agit toutefois là d'un mythe rationalisé, d'un concept boiteux (la culpabilité individuelle découlant d'une faute héréditaire). En outre, cette expression d'une logique de la rétribution ne répond pas à la protestation séculaire découlant du sentiment de la souffrance injustifiable.

Ricœur, à l'instar de Nishitani, se tourne alors vers Kant. La réflexion kantienne évolue également dans le registre de la vision morale: il ne s'agit pas d'établir un système rationnel théorique expliquant le mal, et encore moins une théodicée, mais il s'agit de s'en tenir au niveau pratique qui cherche à comprendre d'où vient que nous faisons le mal. Or, à la notion quasi mythique de péché originel, Kant substitue ici celle de "mal radical» : nul schéma juridico-biologique, mais la notion d'une maxime suprême qui sert de fondement subjectif ultime à toutes les maximes mauvaises. Elle fonde la propension au mal à l'encontre de la prédisposition au bien. Mais la raison d'être de ce mal reste «inscrutable» (unerforschbar).

Or, c'est bien cet inscrutable que - loin de toute théodicée ou de toute autre ontothéologie dialectique - le théologien Karl Barth cherche à interroger. Et Ricœur l'évoque en conclusion de son examen des thèses sur le mal, parlant à son propos de «dialectique brisée ». C'est à la thématique barthienne du néant (das Nichitige) que s'attache ici Ricœur, se rapprochant ainsi singulièrement de Nishitani qui, également, parmi tous les théologiens contemporains avait choisi Barth comme interlocuteur privilégié. Seule une théologie qui renonce à toute totalisation systématique, une théologie «brisée », peut se risquer à penser le mal. Das Nichitige, qui correspond bien au 虚無 (kyomu) nishitanien, désigne une réalité inconciliable avec la bonté de Dieu: c'est un néant, non seulement de privation, mais de corruption et de destruction, source du mal radical autant que de la souffrance injustifiable. Un tel néant est ce qui a été vaincu, dit Barth, non point par une justification ontothéologique, mais par la mort sacrificielle du Christ en croix. Même si fait encore défaut la pleine manifestation de l'élimination du mal, grâce au Christ, nous pouvons continuer à combattre celui-ci en sachant d'une certaine manière qu'il est vaincu d'avance. 
C'est à ce stade que Ricœur souligne alors un flottement de la pensée barthienne lorsque celle-ci cherche à aller au-delà de la christologie et à expliquer le mal comme ce qui existe par la colère divine: il est "ce que Dieu ne veut pas». Comme si Barth cherchait ainsi à sauver la toute-puissance et la toute-bonté de Dieu face à l'inscrutable du mal, comme s'il était tenté malgré tout par une certaine forme de théodicée. Et Ricœur de conclure que toute tentative d'expliquer le mal, théologiquement ou philosophiquement, est un refus d'en reconnaître le caractère irréductiblement aporétique.

Paul Ricœur termine alors sa méditation sur le mal par un geste qui le rapproche singulièrement de l'enseignement bouddhique de la trisiksa (les trois entraînements), selon lequel on ne peut se contenter de la pensée (prajna $)$, pour progresser spirituellement, mais qu'il faut aussi l'action éthique (sîla) et la transformation de soi par la méditation (samādhi): "En conclusion, je voudrais souligner que le problème du mal n'est pas seulement un problème spéculatif: il exige la convergence entre pensée, action (au sens moral et politique) et une transformation spirituelle des sentiments» $(2004,56)$. Sur le plan de la pensée, on l'a vu, le problème du mal est un défi à penser plus et autrement, sans verser dans la tentation des synthèses prématurées et en acceptant les limites imposées par l'aporie... Cette dernière invite, non à une solution, mais à une réponse productive sur le plan de l'action: le mal est ce qui doit être combattu, éthiquement et politiquement. La question théorique de l'origine du mal se renverse ainsi en une tâche pratique à accomplir dans le présent et le futur. Mais l'injustifiable de la souffrance irréductible et invincible ne se satisfait pas d'un agir dont les effets restent limités. Cependant, plutôt que de se complaire dans la lamentation et la plainte, il s'agit de transformer son rapport à la souffrance à l'aide d'une sagesse que Ricœur décrit comme un travail de deuil: il propose d'intégrer l'ignorance impliquée par l'aporie du mal (pourquoi le mal? je ne sais pas); il propose d'assumer l'idée que les raisons de croire en Dieu n'ont rien de commun avec le besoin d'expliquer l'origine de la souffrance (aimer Dieu pour rien, comme le suggère la finale du Livre de Job); il propose d'avancer sur le chemin qui renonce à la plainte elle-même et voir peut-être dans la douleur une valeur éducative et purgative, pressentir que Dieu lui-même souffre... Tout ceci présuppose de progresser dans le renoncement aux désirs (celui d'être récompensé pour ses vertus, celui d'être épargné par la souffrance, d'être épargné par la mort...). Et de conclure: "Peut-être cet horizon de la sagesse, dans l'Occident judéo-chrétien, recoupe-t-il celui de la sagesse bouddhique en quelque 
point que seul un dialogue prolongé entre judéo-christianisme et bouddhisme pourrait identifier» $(2004,65)$.

Ainsi Ricœur lui-même, au terme de sa méditation sur la condition souffrante et pécheresse de l'homme, pressent-il qu'elle pourrait se poursuivre dans un dialogue avec le bouddhisme. Et nous venons de constater que, par son souci de rejeter la tentation de la théodicée afin de rendre justice à la dimension ténébreuse du mal, les thèmes et les auteurs qui retiennent son attention sont les mêmes que pour Nishitani, en des commentaires au final assez proches. Pour Ricœur, l'inscrutable du mal nous incite à dépasser le plan de la pensée pour nous engager dans l'action et dans la quête de sagesse. Pour Nishitani, l'épreuve insensée du mal, et du néant dont il est l'occasion, nous invite à consentir à ce dernier et à le laisser se convertir en une vacuité qui permettra de transfigurer notre manière d'être et d'agir - l'action devenant alors spontanément altruiste.

Cette convergence en un point si essentiel, entre Ricœur et Nishitani, nous suggère sans doute de relativiser la portée des divergences quant à la position métaphysique de départ: subjectivité réflexive d'un côté et nonego (無我, muga) de l'autre, voire personnalité et "personnelle impersonnalité »(人格的な非人格性, jinkakutekina hijinkakusei), ne sont-ils peut-être que des modes d'approche et d'expression de postures métaphysiques dont on pourrait découvrir, par un examen plus approfondi, qu'elles sont en vérité conciliables, voire complémentaires.

Revenons pour terminer à l'affirmation originaire que Ricœur érige contre les philosophies notamment existentielles de la négativité, au premier rang desquelles il place Sartre. Il est intéressant de noter à ce propos que Nishitani, pour qui le fondement est un néant apparemment inverse de toute affirmation originaire, cherche à définir ce néant également par opposition au même Sartre qu'il critique longuement (36-41): signe qu'il ne s'agit pas chez lui d'une négativité identique, mais bien d'une ouverture que le creusement de la béance du néant par l'éclaircie de la vacuité permettra amplement de commenter. Ricœur et Nishitani se rejoignent ainsi pour dire que l'énigme de la négativité du mal est plus qu'un défi intellectuel, c'est une épreuve existentielle qui invite à une métamorphose intime par laquelle peut se dévoiler une nouvelle plénitude.

\section{Références}

NishitAni, K. 西谷啓治 (1961), Shūkyō to wa nanika 宗教とは何か, Tōkyō 東京, Sōbunsha 創文社. 
Ricceur, P. (I955), « Négativité et affirmation originaire ", Histoire et vérité, Paris, Seuil, p. 336-360.

(1969), "La question du sujet », Le conflit des interprétations, Paris, Seuil, p. 233-261.

(2004), Le mal. Un défi à la philosophie et à la théologie, Genève, Labor et Fides.

\section{Résumé}

Il s'agit d'un exposé de la manière dont Nishitani présente la question du mal et du péché dans Qu'est-ce que la religion?, tâchant de montrer que cette question joue un rôle essentiel dans l'approfondissement de la prise de conscience en direction du néant et de la vacuité. Afin de mieux mettre en perspective la pensée de Nishitani, celle-ci est brièvement comparée à celle de Paul Ricœur à propos de la même problématique. La mise en relief de leurs positions métaphysiques divergentes permet de constater que le "penser plus et autrement ", qu'induit l'aporie du mal, les conduit à des positions finalement très proches.

\section{Abstract}

This paper offers an account of the way Nishitani presents the problem of evil and sin in Religion and Nothingness, trying to prove that this question has an essential role in the deepening of self-awareness towards nothingness and emptiness. In order to see Nishitani's thought in a wider horizon, it is briefly compared to Ricour's analysis of the same problem. One can then conclude that, despite the differing metaphysical standpoints of the two philosphers, they tend to agree on the question of evil because of its aporetic nature, forcing to "think more an differently». 